\title{
Propyl Gallate Inhibits Adipogenesis by Stimulating Extracellular Signal-Related Kinases in Human Adipose Tissue-Derived Mesenchymal Stem Cells
}

\author{
Jeung-Eun Lee', Jung-Min Kim', Hyun-Jun Jang ${ }^{1,2}$, Se-young Lim', Seon-Jeong Choi', Nan-Hee Lee ${ }^{4}$, \\ Pann-GhillSuh', and Ung-Kyu Choi ${ }^{\beta, *}$
}

\begin{abstract}
Propyl gallate (PG) used as an additive in various foods has antioxidant and anti-inflammatory effects. Although the functional roles of $P G$ in various cell types are well characterized, it is unknown whether PG has effect on stem cell differentiation. In this study, we demonstrated that PG could inhibit adipogenic differentiation in human adipose tissue-derived mesenchymal stem cells (hAMSCs) by decreasing the accumulation of intracellular lipid droplets. In addition, PG significantly reduced the expression of adipocyte-specific markers including peroxisome prolif-

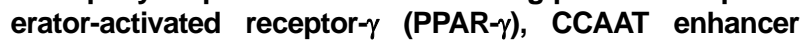
binding protein- $\alpha(C / E B P-\alpha)$, lipoprotein lipase (LPL), and adipocyte fatty acid-binding protein 2 (aP2). PG inhibited adipogenesis in hAMSCs through extracellular regulated kinase (ERK) pathway. Decreased adipogenesis following $P G$ treatment was recovered in response to ERK blocking. Taken together, these results suggest a novel effect of PG on adipocyte differentiation in hAMSCs, supporting a negative role of ERK1/2 pathway in adipogenic differentiation.
\end{abstract}

\section{INTRODUCTION}

Propyl gallate (PG, 3,4,5-trihydroxybenzoic acid propyl ester, Fig. 1A), an ester formed by the condensation of gallic acid with n-propanol, is used as an antioxidant in a wide range of foods cosmetics, hair products, adhesives, lubricants, food packing materials, pharmaceuticals, and personal hygiene products (Han et al., 2010). PG prevents fat oxidation as well as other

\footnotetext{
${ }^{1}$ School of Nano-Bioscience and Chemical Engineering, Ulsan National Institute of Science and Technology, Ulsan 689-798, Korea, ${ }^{2}$ Division of Molecular and Life sciences, Pohang University of Science and Technology, Pohang, Korea, ${ }^{3}$ Department of Food Science and Technology, Korea National University of Transportation, Chungbuk 368-701, Korea ${ }^{4}$ Department of Food Nutrition and Cook, Daegu Science University, Daegu 702-723, Korea

*Correspondence: ukchoi@ut.ac.kr
}

Received 2 September, 2014; revised 23 December, 2014; accepted 24 December, 2014; published online 26 March, 2015

Keywords: adipogenesis, human adipose tissue-derived mesenchymal stem cells, propyl gallate synthetic antioxidants such as butylated hydroxytoluene (BHT), butylated hydroxyanisole (BHA), tert-butyl hydroquinone (TBHQ), and ethylene diaminetetraacetic acid (EDTA) (Foti et al., 2010). In the food industry, PG is added to vegetable oil, mayonnaise, meat, soups, milk powder, spices, candies, snacks, vitamins, and chewing gums. It is practically used as a food additive (particularly in oils and fats). It is authorized for use in Korea and many others countries (Daniel, 1986). Because of its prevalent usage, the potential toxicity of PG has been investigated in vivo (Dacre, 1974; Wu et al., 1994) and in vitro to assess its various properties, including cytogenetic effects (Abdo et al., 1986) and mutagenicity (Rosin and Stich, 1980).

Overweight and obesity defined as abnormal or excessive fat accumulation are major risk factors of several metabolic diseases (such as type II diabetes, cardiovascular diseases, hypertension) and certain cancers (Park et al., 2011). Obesity occurs when the calories ingested exceed the energy used. The excess calories end up in fat cells in the form of triglycerides (Spiegelman and Flier, 2001). Obesity causes dysfunctional production of adipokines and induces pro-inflammatory signaling pathways, leading to increase of inflammatory biomarkers (Hotamisligil et al., 1993). Therefore, research has been conducted to identify novel factors with obesity-fighting effects from natural compounds (Bin and Choi, 2012).

Mesenchymal stem cells (MSCs) are a group of multi-potent adult stem cells that can be isolated from various tissues, including bone marrow, smooth muscle, adipose tissue, skeletal muscle, trabecular bone, articular cartilage, umbilical cord blood, liver, spleen, and fetal tissue (Bianco et al., 2008; Chen et al., 2006; Lim et al., 2011; Mimeault and Batra, 2008). MSCs have several generations of self-renewal ability while maintaining their capacity to differentiate into multiple tissues. MSCs can be differentiated into the same mesenchymal lineage cells (such as osteocytes, chondrocytes, and adipocytes) and cells of other lineages such as neuronal and liver cells (Dezawa et al., 2004; 2005; Pan et al., 2008; Pittenger et al., 1999). Because of these properties, MSCs have become an interesting target for potential therapeutic use in regenerative medicine and tissue engineering. Therefore, identifying factors that regulate MSCs differentiation is essential to use these cells in regenerative medicine.

Recent studies have shown that several compounds can modulate adipogenic differentiation by regulating MAPK activity, highlighting the central roles of MAPKs in adipocyte differentiation. All three MAP kinases have been reported to be involved 
in adipogenesis of hMSCs, with phosphorylation of ERK and JNK as negative regulator, whereas phosphorylation of p38 as positive regulator (Tominaga et al., 2005). PPAR- $\gamma$ is a critical adipogenesis transcription factor (Rosen et al., 2000; Spiegelman, 1998). However, the effect of PG on stem cell differentiation remains unknown. In addition, it was unknown whether PG could activate MAPKs in adipogenesis of hMSCs. Therefore, the objectives of this study were: 1) examine the effect of $P G$ on adipogenic differentiation of hAMSCs; 2) determine whether PG could activate MAPKs.

\section{MATERIALS AND METHODS}

\section{Materials}

PG, 3-isobutylmethylxanthine, dexamethasone, rosiglitazone, indometacin, insulin, methyl thiazolyltetrazolium (MTT), and Oil Red-O were purchased from Sigma-Aldrich (USA). Antibodies against CCAAT enhancer binding protein- $\alpha$, peroxisome proliferator-activated receptor-activated receptor- $\gamma$, ERK2, adipsin, and aP2 antibodies were obtained from Santa Cruz Biotechnology (USA). Antibodies against p-ERK1/2, p-p38, and p-JNK were purchased from Cell Signaling Technology (USA). Actin antibody was purchased from MP Biomedicals (USA). Antimouse and anti-rabbit horseradish peroxidase-conjugated secondary antibodies were obtained from KPL (USA). PD98059 was purchased from Calbiochem (USA).

\section{Cell culture}

Subcutaneous adipose tissues were obtained from women patients (mean age, 28 years) undergoing elective surgery after obtaining informed consent. hAMSCs were isolated from adipose tissue according to a method described previously (Lee et al., 2004) with slight modifications. Briefly, hAMSCs were maintained in growth medium (GM) consisting of $\alpha$-MEM supplemented with $10 \%$ fetal bovine serum (FBS), $100 \mathrm{U} / \mathrm{ml}$ penicillin, and $100 \mathrm{mg} / \mathrm{ml}$ of streptomycin (Gibco BRL, USA). Passages 3 to 10 were used in experiments.

\section{Adipogenic induction}

hAMSCs were grown to confluence before differentiation induction. Adipogenic differentiation was induced by culturing the hAMSCs for 14 days in adipogenic induction medium (AIM, 10\% FBS, $1 \mu \mathrm{M}$ dexamethasone, $500 \mu \mathrm{M}$ 3-isobutyl-1-methylxanthine, $1 \mu \mathrm{M}$ insulin, and $100 \mu \mathrm{M}$ indomethacin in $\alpha-\mathrm{MEM})$. After 6 days, cells were cultured in AIM containing only $10 \%$ FBS and $1 \mu \mathrm{M}$ insulin in $\alpha$-MEM. AIM was changed every 3 days.

\section{Oil Red-O staining}

Differentiated adipocytes were stained with Oil Red-O as an indicator of intracellular lipid accumulation. Cells were cultured in AIM as described above for 14 days. Lipid droplets within differentiated adipocytes from hAMSCs were observed using Oil Red-O staining method with modification. Briefly, cells were washed twice with phosphate buffered saline (PBS) and fixed with $4 \%$ paraformaldehyde for $2 \mathrm{~h}$ at $4^{\circ} \mathrm{C}$. After two washes in PBS, cells were stained for $2 \mathrm{~h}$ in freshly diluted Oil Red-O solution (six parts of Oil Red-O stock solution and four parts of $\mathrm{H}_{2} \mathrm{O}$ ). The Oil Red-O stock solution was $0.5 \%$ Oil Red-O in isopropanol at $4^{\circ} \mathrm{C}$. The stain was then removed. Cells were washed twice with PBS. Images of cells stained with Oil Red-O were obtained with an Olympus IX 71 microscope equipped with an Olympus DP72 camera (Japan). For quantitative analysis of Oil Red-O staining, cells were exposed to isopropanol for $30 \mathrm{~min}$. Absorbance value of the supernatant was measured at
$540 \mathrm{~nm}$ using a Biotrak II plate reader (Amersham Biosciences, Biochrom Ltd., Cambridge England).

\section{MTT assay}

hAMSCs were seeded at a density of $1 \times 10^{4}$ cells/well into a 96-well plate and cultured for appropriate length of time as indicated. MTT was dissolved in PBS at $5 \mathrm{mg} / \mathrm{ml}$. The stock solution was filtered and added to the culture medium at a final concentration of $0.5 \mathrm{mg} / \mathrm{ml}$. The plates were incubated at $37^{\circ} \mathrm{C}$ for $2 \mathrm{~h}$. Dark brown formazan crystals formed after reduction of tetrazolium by mitochondria in living cells. The formazan crystals were dissolved in dimethylsulfoxide (DMSO) and the absorbance values of the samples were measured at $540 \mathrm{~nm}$.

RNA extraction and real-time polymerase chain reaction (RT-PCR)

Total RNA was extracted from hAMSCs using TRlzol reagent (Invitrogen, USA). cDNA was reverse-transcribed from $1.5 \mu \mathrm{g}$ total cellular RNA using oligo(dT) primers and Moloney Murine Leukemia Virus reverse transcriptase (Promega Corp., USA). cDNA was amplified using the following primers: peroxisome proliferatoractivated receptor gamma (PPAR- $\gamma$ ); CCAAT-enhancer-binding proteins (C/EBP)- $\alpha$ 5'-AACCTTGTGCCTTGGAAATG-3' (sense), 5'-CCTGCTCCCCTCCTTCTCT-3' (antisense); adiponectin 5'ACCACTATGATGGCTCCACT-3' (sense), 5'-GGTGAAGAGCATAGCCTTGT-3' (antisense); fatty acid binding protein (aP2) 5'-AACCTTAGATGGGGGTGTCCTG-3' (sense), 5'-TCGTGGAAGTGACGCCTTTC-3' (antisense); lipoprotein lipase (LPL) 5'-CTGGACGGTAACAGGAATGTATGAG-3' (sense), 5'-CATCAGGAGAAAGACGACTCGG-3' (antisense), and the reference gene, ribosomal protein large P0 (RPLP0) 5'-GGAATGTGGGCTTTGTGTTC-3' (sense), 5'-TGCCCCTGGAGATTTTAGTG-3' (antisense). For real-time PCR, total RNA (100 ng) was amplified with One Step SYBR RT-PCR kit using a Light Cycler 2.0 PCR system (Roche Diagnostics, Manheim, Germany). PCR conditions consisted of a $10-\mathrm{min}$ hot start at $95^{\circ} \mathrm{C}$, followed by 45 cycles of $15 \mathrm{~s}$ at $95^{\circ} \mathrm{C}, 10 \mathrm{~s}$ at $60^{\circ} \mathrm{C}$, and $30 \mathrm{~s}$ at $72^{\circ} \mathrm{C}$. mRNA expression levels were normalized against RPLPO expression. The experiment was repeated twice.

\section{Western blot analysis}

Whole cell lysates were prepared in lysis buffer $(1 \%$ Triton $X$ $100,10 \%$ glycerol, $150 \mathrm{mM} \mathrm{NaCl}, 50 \mathrm{mM}$ HEPES, pH 7.3, 1 mM EGTA, $1 \mathrm{mM}$ sodium orthovanadate, $1 \mathrm{mM}$ sodium fluoride, $1 \mathrm{mM}$ phenylmethysulfonyl fluoride, $10 \mathrm{mg} / \mathrm{ml}$ leupeptin, and 10 $\mathrm{mg} / \mathrm{ml}$ aprotinin). Lysates were then centrifuged at $14,000 \mathrm{rpm}$ for $15 \mathrm{~min}$ at $4^{\circ} \mathrm{C}$. Electrophoresis was performed using 8 12\% polyacrylamide gels. After proteins were electro-transferred to nitrocellulose membranes, they were incubated with primary antibodies overnight at $4^{\circ} \mathrm{C}$. After incubation with appropriate peroxidase conjugated secondary antibody followed by washes, protein bands were detected with enhanced chemiluminescence system (ECL System, Amersham).

\section{Plasmids and luciferase activity assay}

Plasmid 3-x PPRE-tk-LUC containing three copies of PPAR- $\gamma$ response element and pSV-PPAR- $\gamma 2$ was used (Seo et al., 2004). The pRL-SV40 construct, the Renilla luciferase expression vector, was purchased from Promega Corp. Human embryonic kidney 293 (HEK293) cells were seeded into 24-well plates and cultured for $24 \mathrm{~h}$ before transfection. A DNA mixture containing the PPRE-luciferase reporter plasmid $(0.1 \mu \mathrm{g})$, pSVPPAR- $\gamma 2(0.1 \mu \mathrm{g})$, and an internal control plasmid pRL-SV-40 (25 ng) was transfected using Lipofectamine transfection rea- 
$\boldsymbol{A}$<smiles>CCCOC(=O)c1cc(O)c(O)c(O)c1</smiles>

Propyl gallate

B
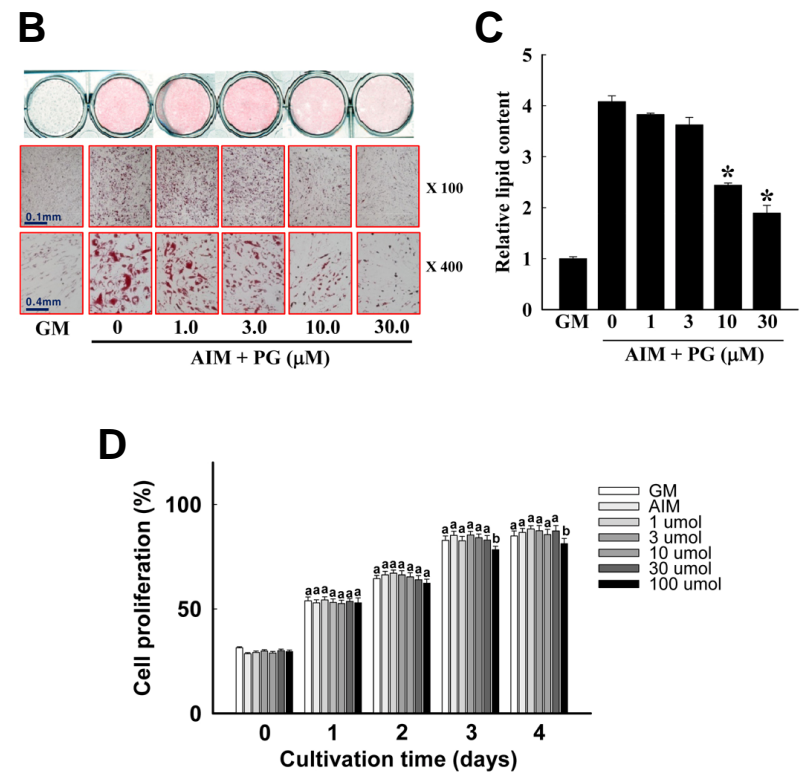

Fig. 1. Propyl gallate (PG) inhibits lipid accumulation in hAMSCs. (A) Chemical structure of PG; (B) Confluent hAMSCs were cultured in GM or AIM with various concentrations of PG. The media were changed every 3 days. Cells were stained with Oil Red-O on day 14 Red staining indicated the presence of neutral lipids. Images are representative of experiment performed in triplicates; (C) For quantitative analysis, Oil Red-O staining was quantified by exposing the cells to isopropanol for $30 \mathrm{~min}$. The absorbance of the supernatant was measured at $540 \mathrm{~nm}$ using a Biotrak II plate reader. Mean values were obtained from day 14 with the value of GM-cultured cells as 1.0. Other values were normalized against the value of GMcultured cells. Values are presented as mean \pm SD. Error bars indicated the range of results. Experiment was performed in triplicates. Results were representative of three independent experiments (magnification $\times 100)\left({ }^{*} p<0.05\right)$; (D) Confluent hAMSCs were cultured in GM or AIM with or without 1 100 $\mu \mathrm{M}$ PG. Cells were harvested at the indicated times. Cell viability was examined by MTT assay. Values are presented as mean \pm SD. Error bars indicate the range of results from experiment performed in triplicates $\left({ }^{*} p<0.05\right)$. Results are representative of three independent experiments. AIM: adipogenic induction medium; GM: growth medium; hAMSCs: human adipose tissue-derived mesenchymal stem cells; MTT: methyl thiazolyltetrazolium; SD: standard deviation.

gent according to the manufacturer's recommendations. At 24 $\mathrm{h}$ post transfection, cells were incubated for an additional $36 \mathrm{~h}$ following treatment with positive control or test materials. Luciferase activity of cell lysates was measured using the DualLuciferase Reporter Assay System (Promega) according to the manufacturer's instructions. Relative luciferase activity was normalized to transfection efficiency using the corresponding
Renilla luciferase activity.

\section{Statistical analysis}

Data were expressed as mean \pm standard deviation (SD) of three or more independent experiments. Statistical significance was estimated by Student's $t$-test. Differences were considered significant when $p$ value was less than 0.05 . All statistical tests were performed using SPSS for Windows version 18.0 (SPSS, USA).

\section{RESULTS}

\section{PG reduces lipid droplet formation}

hAMSCs were treated with AIM in the presence of PG to investigate the effect of PG on adipocyte differentiation. At 14 days after the initiation of differentiation, the accumulation of lipid droplets, a characteristic of the adipocyte formation process, was monitored using Oil Red-O staining. Major changes were observed in PG-treated hAMSCs compared to vehicle controltreated hAMSCs under adipogenic differentiation conditions. Treatment with PG significantly $(p<0.05)$ reduced lipid droplets accumulation and adipocyte differentiation of hAMSCs in a dose-dependent manner (Fig. 1B). To further validate these phenotypic results, we measured the neutral lipid content in these cells and found that the neutral lipid content was significantly $(p<0.05)$ decreased following PG treatment (Fig. 1C). These results indicated that PG suppressed adipogenesis in hAMSCs. In addition, we performed MTT assay to determine whether PG had cellular toxicity. MTT assay results revealed that PG did not affect the viability of cells cultured in AIM with up to $30 \mu \mathrm{M} P G$ (Fig. 1D). Taken together, these results suggest that $P G$ could inhibit adipocyte differentiation without affecting cellular toxicity.

PG decreases mRNA levels and protein expression of adipogenic markers

Adipocyte differentiation is accompanied by altered expression of various transcription factors and adipocyte-specific genes (Sermeus et al., 2008). To further confirm the effect of PG on adipocyte differentiation, we performed real-time PCR to determine the expression of PPAR- $\gamma$ and C/EBP- $\alpha$, two key transcriptional factors involved in adipogenesis. We also checked the mRNA expression levels of aP2, adiponectin, and LPL. The mRNA expression levels of PPAR- $\gamma, \mathrm{C} / \mathrm{EBP}-\alpha, \mathrm{LPL}$, aP2, and adiponectin were significantly $(p<0.05)$ decreased following treatment with $10 \mu \mathrm{M}$ PG (Fig. 2A). At 6 days after PG treatment, the mRNA expression levels of both PPAR- $\gamma$ and C/EBP$\alpha$ expressions were significantly $(p<0.05)$ inhibited. The transcriptional levels of aP2, adiponectin, and LPL were also significantly $(p<0.05)$ inhibited by PG treatment. In addition, the protein levels of adipocyte-specific markers were significantly ( $p$ $<0.05$ ) decreased following PG treatment under adipogenic differentiation conditions (Fig. 2B). These results suggest that PG can inhibit adipocyte differentiation of hAMSCs.

PG inhibits PPAR- $\gamma$ activity

PPAR- $\gamma$ is a critical adipogenes is transcription factor (Rosen et al., 2000; Spiegelman, 1998). Some natural products can regulate adipogenesis by modulating PPAR- $\gamma$ activity (Gong et al., 2009; Saito et al., 2009; Shang et al., 2007). Based on these reports, we tested whether PG could inhibit adipocyte differentiation by regulating PPAR- $\gamma$ activity. Because the transfection efficiency of hAMSCs was very low, we used HEK293 cells to examine the effect of PG on PPAR- $\gamma$ activity. HEK293 cells were 
A
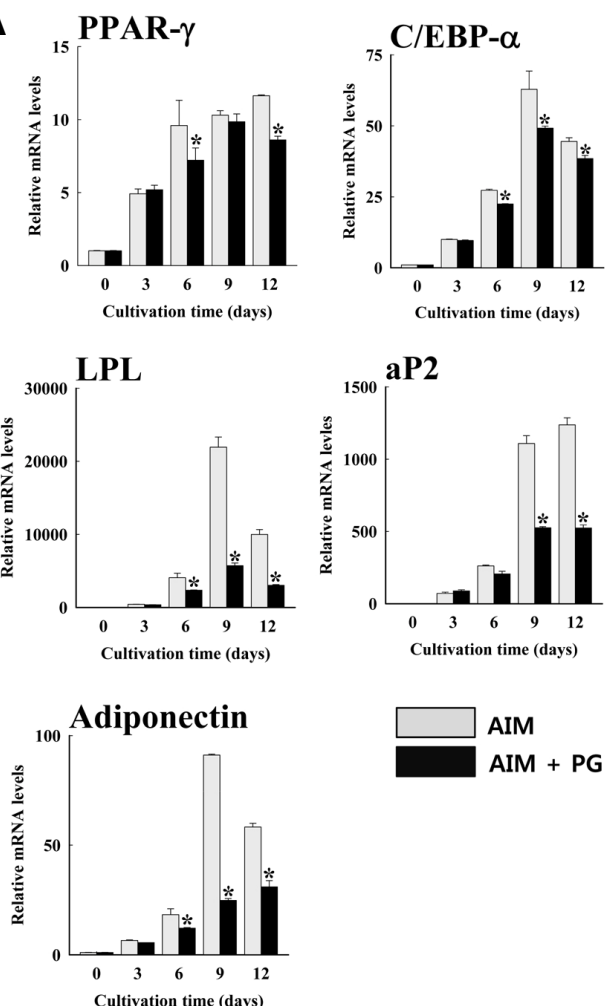

B

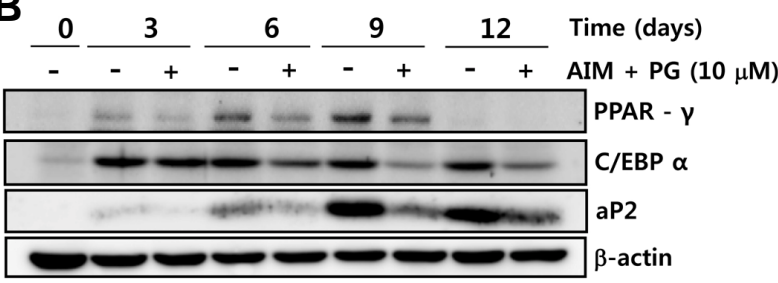

Fig. 2. Propyl gallate $(P G)$ decreases $m R N A$ and protein level of adipocyte-specific markers. (A) hAMSCs were cultured in AIM with or without $10 \mu \mathrm{M} \mathrm{PG}$. Gene expression of C/EBP- $\alpha$, PPAR- $\gamma$, LPL, aP2, and adiponectin was examined by real-time PCR. The mean values obtained from vehicle-treated cells at 0 day were considered as 1.0. Others were relative values. Values are presented as mean \pm SD. Error bars indicate results range for treatment performed in triplicates. ${ }^{*} p<0.05$ compared to control. Results are representative of two independent experiments; (B) PG decreases expression of adipocyte-specific marker proteins. Protein levels of adipogenic markers were examined during adipogenesis. Results are representative of three independent experiments. aP2: adipocyte fatty acid-binding protein; C/EBP- $\alpha$ : CCAAT enhancer binding protein- $\alpha$ LPL, lipoprotein lipase; PCR: polymerase chain reaction; PPAR- $\gamma$ : peroxisome proliferator-activated receptor- $\gamma$.

co-transfected with a PPREx3-tk-luciferase reporter plasmid and PPAR- $\gamma 2$ expression vector. We used rosiglitazone, a synthetic PPAR- $\gamma$ ligand, as a positive control. At $36 \mathrm{~h}$ post treatment, rosiglitazone $(100 \mathrm{nM})$ significantly $(p<0.05)$ increased the activity of PPAR- $\gamma$ (Fig. 3). Co-treatment with PG reduced the transcriptional activity of PPAR- $\gamma$ in a dose-dependent manner (Fig. 3). These results suggest that PG can suppress adipogenesis of

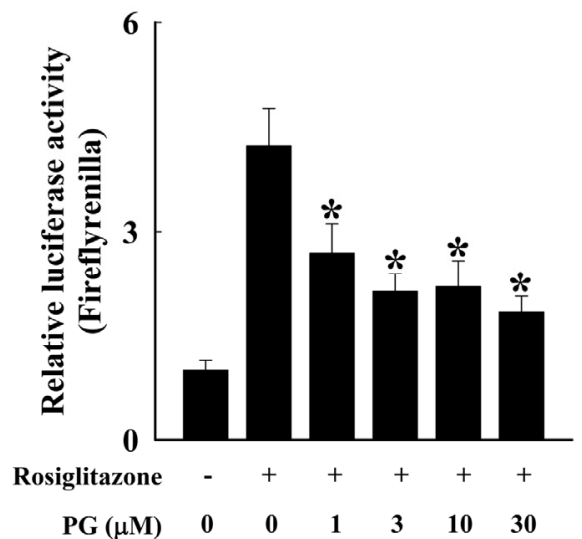

Fig. 3. Propyl gallate (PG) inhibits PPAR- $\gamma$ activity. HEK293 cells were co-transfected with PPREx3-tk-luciferase reporter plasmid, the Renilla luciferase plasmid, and the PPAR- $\gamma 2$ expression vector. At $12 \mathrm{~h}$ post transfection, cells were treated with $100 \mathrm{nM}$ rosiglitazone and $P G$ for $36 \mathrm{~h}$. Ligand-dependent transcriptional activity was measured by dual-luciferase assay. Data are presented as the ratio of firefly luciferase activity to Renilla luciferase activity. Values are presented as mean \pm SD. ${ }^{*} p<0.05$ compared to control; PPAR- $\gamma$ peroxisome proliferator-activated receptor- $\gamma$.

hAMSCs by inhibiting PPAR- $\gamma$ activity.

\section{PG negatively regulates adipogenic differentiation by activating ERK}

Recent studies have shown that several compounds can modulate adipogenic differentiation by regulating MAPK activity, highlighting the central roles of MAPKs in adipocyte differentiation. Based on these reports, we determined whether PG could activate MAPKs in hAMSCs. In serum starvation condition, PG (10 $M)$ induced ERK1/2 and p38 phosphorylation, but not JNK phosphorylation (Fig. 4A). In addition, treatment with PG (10 M) potentiated ERK1/2 and p38 phosphorylation under AIM conditions compared to treatment with vehicle control (Figs. 4B and 4C).

To determine whether PG could inhibit adipogenesis via the ERK or p38 signaling pathway, we used the specific mitogenactivated protein kinase (MEK) inhibitor PD98059 to inhibit ERK phosphorylation. MEK is an upstream kinase of ERK. ERK activation can be prevented by blocking MEK activity(Crews et al., 1992). SB203580 was used to prevent p38 activation. PGinduced ERK $1 / 2$ and $\mathrm{p} 38$ phosphorylation was significantly $(p<$ 0.05 ) inhibited in response to treatment by each inhibitor (Fig. 5A). Treatment of PD98059 recovered PG-mediated inhibition of lipid accumulation in a dose-dependent manner (Fig. 5B), indicating that $P G$ prevented adipogenic differentiation of hAMSCs via the ERK pathway. The absorbance of extracted Oil Red-O in the presence of PD98059 $(20 \mu \mathrm{M})$ recovered to $77 \%$ of that found under normal differentiating conditions (Fig. 5B). In contrast, inhibiting the p38 signal pathway with the specific inhibitor SB203580 did not affect PG anti-adipogenic activity (Fig. 5B). Additionally, expressions of the adipocyte specific protein aP2 and adipsin were rescued in a dose dependent manner by inhibiting ERK (Fig. 5C). Our data clearly show that the ERK signaling pathway is required for anti-adipogenic activity of PG. Taken together, these results suggest that PG inhibits adipogenesis possibly via sustained activation of ERK without depending on the activation of other MAPKs. 
A

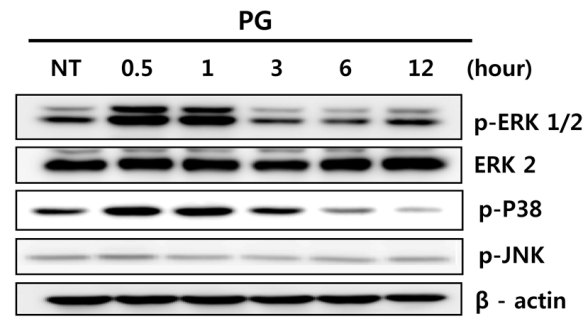

B

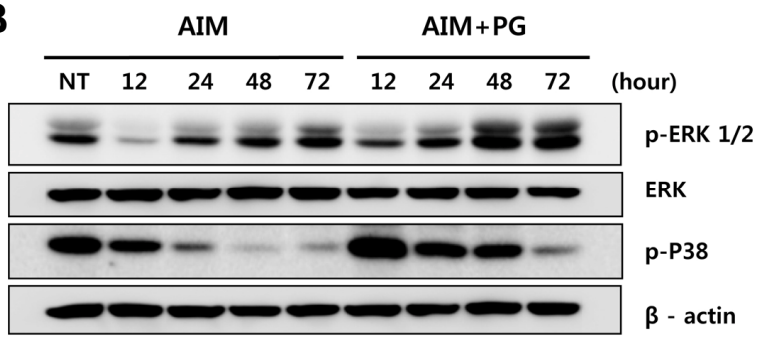

C

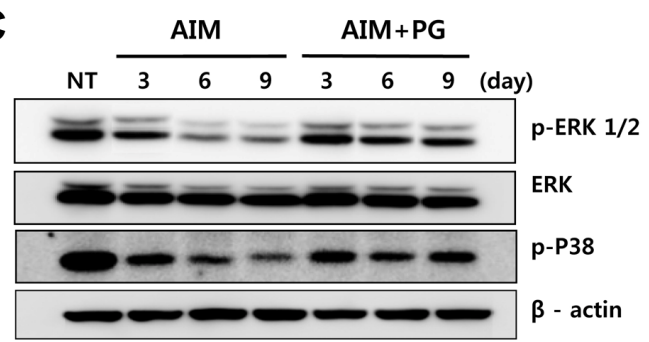

Fig. 4. Propyl gallate (PG) activates phosphorylation of ERK $1 / 2$ and p38 in hAMSCs. (A) hAMSCs were treated with $10 \mu \mathrm{M}$ PG under serum-free conditions. Cell lysates were prepared at different time points as indicated. ERK1/2, p38, and JNK1 phosphorylation was examined by immunoblotting; (B) hAMSCs were incubated with or without PG $(10 \mu \mathrm{M})$ in AIM. Cell lysates were subjected to immunoblot analysis. ERK1/2 and p38 phosphorylation was stimulated by PG treatment; (C) hAMSCs were treated with or without PG $(10 \mu \mathrm{M})$ under adipogenic differentiation condition. ERK1/2 and p38 phosphorylations as well as ERK2 and $\beta$-actin expression were examined. $\beta$-actin was used as loading control. ERK: extracellular regulated kinase; JNK: Jun-kinase; hAMSCs: human adipose tissue-derived mesenchymal stem cells; AIM: adipogenic induction medium.

\section{DISCUSSION}

PG has been used as a synthetic antioxidant in processed food, cosmetics, and food packaging materials to prevent rancidity and spoilage. It has also been used to preserve and stabilize medicines. Previous studies on PG have focused on its antioxidant and anti-inflammatory effects. Several studies have revealed the benefits of PG as antioxidant (Chen et al., 2007; Raghavan and Hultin, 2005; Reddan et al., 2003; Wu et al., 1994), chemopreventive agent (Hirose et al., 1993; Karthikeyan et al., 2005), and anti-inflammatory agent (Jeon and Kim, 2007). However, the potential effect of PG on stem cell differentiation has not been studied yet previously. In this study, we focused on the activity of PG during hAMSC adipogenic differentiation. PG significantly decreased adipocyte differentiation of hAMSCs and 3T3-L1 cells (Supplementary Fig. 1) without cellular toxicity.
A
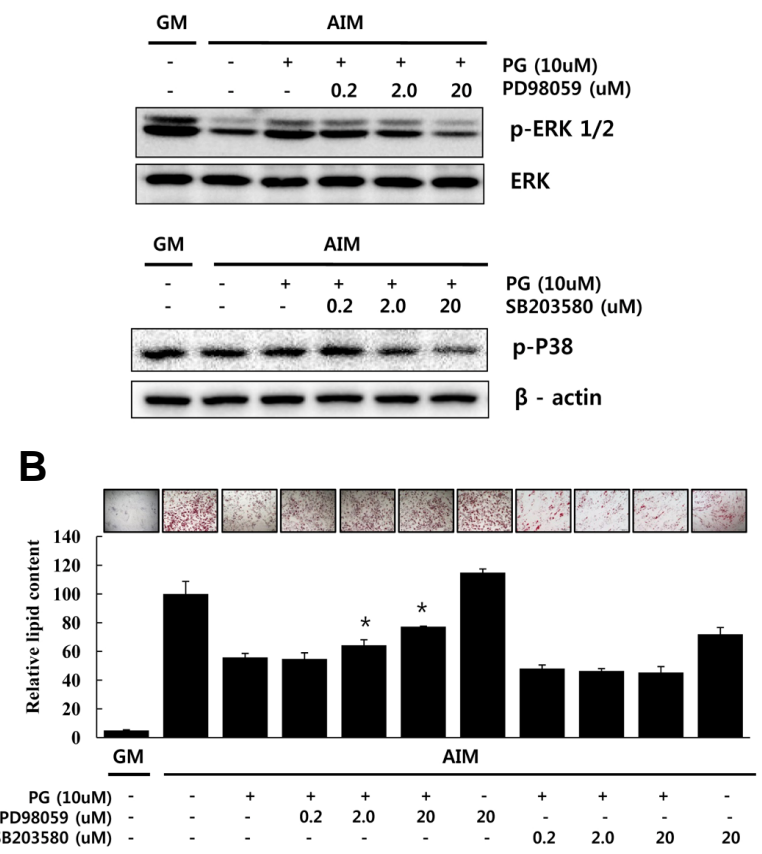

C

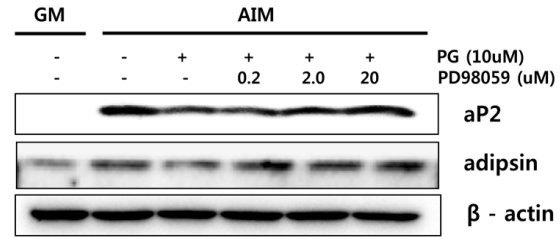

D

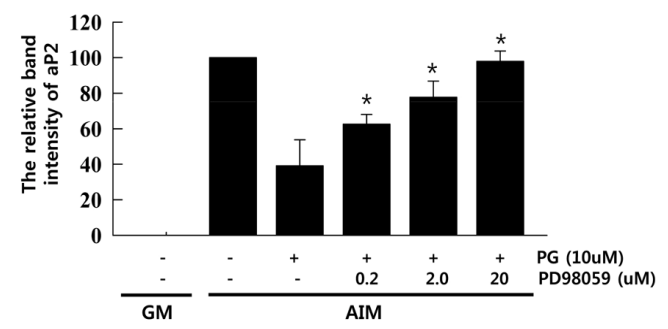

Fig. 5. Propyl gallate (PG) negatively regulates adipogenesis through ERK activation. (A) hAMSCs were pre-treated with vehicle or PD98059 for $1 \mathrm{~h}$ before the addition of AIM containing $10 \mu \mathrm{M} \mathrm{PG}$. ERK $1 / 2$ phosphorylation was monitored by immunoblotting $30 \mathrm{~min}$ after PG treatment. hAMSCs were pre-treated with vehicle or SB203580 for $1 \mathrm{~h}$ before the addition of AIM containing $10 \mu \mathrm{M} \mathrm{PG}$. p38 phosphorylation was monitored by immunoblotting 30 min after the PG treatment; (B) hAMSCs were cultured under adipogenic differentiation conditions with or without $P G(10 \mu \mathrm{M})$. The effect of PD98059 or SB203580 was examined. Lipid accumulation was confirmed by Oil Red-O staining at 14 days after adipogenic induction. Values represent the mean $\pm S D$; (C) The expression of adipogenic markers aP2 and adipsin was examined in PG-treated cells with or without treatment with PD98059; (D) aP2 protein levels in hAMSCs were examined at day 14 after adipogenic induction. The levels of aP2 were normalized to actin. hAMSCs: human adipose tissue-derived mesenchymal stem cells; AIM: adipogenic induction medium; ERK: extracellular regulated kinase. 
Our results showed that $P G$ inhibited adipocyte differentiation of hAMSCs and lipid droplet formation. Adipogenic marker expression was suppressed during adipogenesis of hAMSCs in response to PG treatment. In addition, PG reduced PPAR- $\gamma$ activity. To our knowledge, this is the first report to demonstrate an effect of $P G$ on stem cell differentiation.

Many studies have shown that several compounds could negatively regulate adipocyte differentiation. Genistein, an isoflavone derived from soybean, has been reported to be able to suppress adipogenic differentiation of hAMSCs through the Wnt/ $\beta$-catenin signaling pathway (Kim et al., 2010b). Wedelolatone, an herbal medicine used to treat septic hick, has been reported to be capable of inhibiting adipogenic differentiation in bone marrow stromal cells by activating ERK and JNK (Lim et al., 2012). In addition, some flavonoids such as vitexin, orientin, and lovastatin have been reported to be able inhibit adipogenic differentiation by suppressing PPAR- $\gamma$ expression in bone marrowderived mesenchymal cell cultures (Kim et al., 2010a; Li et al., 2003). Our results suggest a novel effect of $P G$ during adipogenesis in hAMSCs. In this study, it was found that PG could activate ERK1/2 phosphorylation. Blocking ERK activity rescued the inhibitory activity of PG during adipogenic differentiation. Recently, it has demonstrated that multiple signaling pathways are implicated in adipogenesis (Tominaga et al., 2005). MAPKs are involved in hMSCs adipogenesis, in which ERK and JNK are negative regulators, whereas p38 is a positive regulator (Tominaga et al., 2005). Activated PPAR- $\gamma$ is a key regulator from the beginning of adipogenesis. ERK suppresses the PPAR- $\gamma$ transcriptional activity (Camp and Tafuri, 1997; Hu et al., 1996). Our results revealed that PG treatment could stimulate ERK phosphorylation under adipogenic differentiating conditions, and that treatment with the MEK inhibitor PD98059 reversed the inhibitory effect of PG. These data suggest that PG can suppress adipogenesis of hAMSCs by activating ERK. In addition, PG also decreased PPAR- $\gamma$ activity. S112 phosphorylation of PPAR- $\gamma$ by ERK cascade has been reported to be able to not only inhibit RXR/PPAR heterodimers' genomic action on PPREs, but also inhibit adipocyte differentiation (Camp and Tafuri, 1997). Therefore, activation of the ERK cascade could contribute to the inhibition of PPAR- $\gamma$ genomic action through phosphorylation of PPAR- $\gamma$. The suppression of PPAR- $\gamma$ activity might be mediated by ERK. Taken together, these results suggest that PG can inhibit adipogenesis via ERK pathway and the inhibition of PPAR- $\gamma$.

In conclusion, we demonstrated a novel effect of PG during adipogenic differentiation of hAMSCs. PG inhibited lipid droplet formation and adipogenic marker expression in hAMSCs. Our results suggest that PG can inhibit adipogenesis of hAMSCs through the ERK signaling pathway, supporting a negative role of the ERK pathway in adipogenic differentiation.

Note: Supplementary information is available on the Molecules and Cells website (www. molcells.org).

\section{ACKNOWLEDGEMENTS}

The research was supported by a grant from the Academic Research Program of Korea National University of Transportation in 2014.

\section{REFERENCES}

Abdo, K.M., Huff, J.E., Haseman, J.K., and Alden, C.J. (1986). No evidence of carcinogenicity of D-mannitol and propyl gallate in F344 rats or B6C3F1 mice. Food Chem. Toxicol. 24, 1091-1097. Bianco, P., Robey, P.G., and Simmons, P.J. (2008). Mesenchymal stem cells: revisiting history, concepts, and assays. Cell Stem Cell 2, 313-319.

Bin, H.S., and Choi, U.K. (2012). Myricetin inhibits adipogenesis in human adipose tissue-derived mesenchymal stem cells. Food Sci. Biotechnol. 21, 1391-1396.

Camp, H.S., and Tafuri, S.R. (1997). Regulation of peroxisome proliferator-activated receptor gamma activity by mitogenactivated protein kinase. J. Biol. Chem. 272, 10811-10816.

Chen, F.H., Rousche, K.T., and Tuan, R.S. (2006). Technology Insight: adult stem cells in cartilage regeneration and tissue engineering. Nat. Clin. Pract. Rheumatol. 2, 373-382.

Chen, C.H., Liu, T.Z., Chen, C.H., Wong, C.H., Chen, C.H., Lu, F.J., and Chen, S.C. (2007). The efficacy of protective effects of tannic acid, gallic acid, ellagic acid, and propyl gallate against hydrogen peroxide-induced oxidative stress and DNA damages in IMR-90 cells. Mol. Nutr. Food Res. 51, 962-968.

Crews, C.M., Alessandrini, A., and Erikson, R.L. (1992). The primary structure of MEK, a protein kinase that phosphorylates the ERK gene product. Science 258, 478-480.

Dacre, J.C. (1974). Long-term toxicity study of n-propyl gallate in mice. Food Cosmet. Toxicol. 12, 125-129.

Daniel, J.W. (1986). Metabolic aspects of antioxidants and preservatives. Xenobiotica 16, 1073-1078.

Dezawa, M., Kanno, H., Hoshino, M., Cho, H., Matsumoto, N. Itokazu, Y., Tajima, N., Yamada, H., Sawada, H., Ishikawa, H., et al. (2004). Specific induction of neuronal cells from bone marrow stromal cells and application for autologous transplantation. J. Clin. Invest. 113, 1701-1710.

Dezawa, M., Ishikawa, H., Itokazu, Y., Yoshihara, T., Hoshino, M., Takeda, S., Ide, C., and Nabeshima, Y. (2005). Bone marrow stromal cells generate muscle cells and repair muscle degeneration. Science 309, 314-317.

Foti, C., Bonamonte, D., Cassano, N., Conserva, A., and Vena, G.A. (2010). Allergic contact dermatitis to propyl gallate and pentylene glycol in an emollient cream. Australas. J. Dermatol. $51,147-148$.

Gong, Z., Huang, C., Sheng, X., Zhang, Y., Li, Q., Wang, M.W., Peng, L., and Zang, Y.Q. (2009). The role of tanshinone IIA in the treatment of obesity through peroxisome proliferatoractivated receptor gamma antagonism. Endocrinology 150, 104113.

Han, Y.H., Moon, H.J., You, B.R., Kim, S.Z., Kim, S.H., and Park, W.H. (2010). Propyl gallate inhibits the growth of HeLa cells via caspase-dependent apoptosis as well as a G1 phase arrest of the cell cycle. Oncol. Rep. 23,1153-1158.

Hirose, M., Yada, H., Hakoi, K., Takahashi, S., and Ito, N. (1993). Modification of carcinogenesis by alpha-tocopherol, t-butylhydroquinone, propyl gallate and butylated hydroxytoluene in a rat multi-organ carcinogenesis model. Carcinogenesis 14, 23592364.

Hotamisligil, G.S., Shargill, N.S., and Spiegelman, B.M. (1993). Adipose expression of tumor necrosis factor-alpha: direct role in obesity-linked insulin resistance. Science 259, 87-91.

Hu, E., Kim, J.B., Sarraf, P., and Spiegelman, B.M. (1996). Inhibition of adipogenesis through MAP kinase-mediated phosphorylation of PPARgamma. Science 274, 2100-2103.

Jeon, W.K., and Kim, B.C. (2007). WITHDRAWN: Heme oxygenase1 mediates the anti-inflammatory effect of propyl gallate in LPSstimulated macrophages. Biochem. Biophys. Res. Commun. 361, 645-650.

Karthikeyan, K., Sarala Bai, B.R., Gauthaman, K., and Niranjali Devaraj, S. (2005). Protective effect of propyl gallate against myocardial oxidative stress-induced injury in rat. J. Pharm. Pharmacol. 57, 6773.

Kim, J., Lee, I., Seo, J., Jung, M., Kim, Y., Yim, N., and Bae, K. (2010a). Vitexin, orientin and other flavonoids from Spirodela polyrhiza inhibit adipogenesis in 3T3-L1 cells. Phytother. Res. 24, 1543-1548.

Kim, M.H., Park, J.S., Seo, M.S., Jung, J.W., Lee, Y.S., and Kang, K.S. (2010b). Genistein and daidzein repress adipogenic differentiation of human adipose tissue-derived mesenchymal stem cells via Wnt/beta-catenin signalling or lipolysis. Cell Prolif. 43, 594-605.

Lee, R.H., Kim, B., Choi, I., Kim, H., Choi, H.S., Suh, K., Bae, Y.C., and Jung, J.S. (2004). Characterization and expression analysis of mesenchymal stem cells from human bone marrow and 
adipose tissue. Cell. Physiol. Biochem. 14, 311-324.

Li, X., Cui, Q., Kao, C., Wang, G.J., and Balian, G. (2003). Lovastatin inhibits adipogenic and stimulates osteogenic differentiation by suppressing PPARgamma2 and increasing Cbfa1/Runx2 expression in bone marrow mesenchymal cell cultures. Bone 33, 652659.

Lim, S., Jang, H.J., Kim, J.K., Kim, J.M., Park, E.H., Yang, J.H., Kim Y.H., Yea, K., Ryu, S.H., and Suh, P.G. (2011). Ochratoxin A inhibits adipogenesis through the extracellular signal-related kinasesperoxisome proliferator-activated receptor-gamma pathway in human adipose tissue-derived mesenchymal stem cells. Stem Cells Dev. 20, 415-426.

Lim, S., Jang, H.J., Park, E.H., Kim, J.K., Kim, J.M., Kim, E.K., Yea, K., Kim, Y.H., Lee-Kwon, W., Ryu, S.H., et al. (2012). Wedelolactone inhibits adipogenesis through the ERK pathway in human adipose tissue-derived mesenchymal stem cells. J. Cell. Biochem. 113, 3436-3445.

Mimeault, M., and Batra, S.K. (2008). Recent progress on normal and malignant pancreatic stem/progenitor cell research: therapeutic implications for the treatment of type 1 or 2 diabetes mellitus and aggressive pancreatic cancer. Gut 57, 1456-1468.

Pan, R.L., Chen, Y., Xiang, L.X., Shao, J.Z., Dong, X.J., and Zhang, G.R. (2008). Fetal liver-conditioned medium induces hepatic specification from mouse bone marrow mesenchymal stromal cells: a novel strategy for hepatic transdifferentiation. Cytotherapy 10, 668-675.

Park, J.E., Kim, H.T., Lee, S., Lee, Y.S., Choi, U.K., Kang, J.H., Choi, S.Y., Kang, T.C., Choi, M.S., and Kwon, O.S. (2011). Differential expression of intermediate filaments in the process of developing hepatic steatosis. Proteomics 11, 2777-2789.

Pittenger, M.F., Mackay, A.M., Beck, S.C., Jaiswal, R.K., Douglas R., Mosca, J.D., Moorman, M.A., Simonetti, D.W., Craig, S., and Marshak, D.R. (1999). Multilineage potential of adult human mesenchymal stem cells. Science 284, 143-147.

Raghavan, S., and Hultin, H.O. (2005). Model system for testing the efficacy of antioxidants in muscle foods. J. Agric. Food Chem. 53, 4572-4577.

Reddan, J.R., Giblin, F.J., Sevilla, M., Padgaonkar, V., Dziedzic, D.C., Leverenz, V.R., Misra, I.C., Chang, J.S., and Pena, J.T.
(2003). Propyl gallate is a superoxide dismutase mimic and protects cultured lens epithelial cells from $\mathrm{H} 2 \mathrm{O} 2$ insult. Exp. Eye Res. 76, 49-59.

Rosen, E.D., Walkey, C.J., Puigserver, P., and Spiegelman, B.M (2000). Transcriptional regulation of adipogenesis. Genes Dev. 14, 1293-1307.

Rosin, M.P., and Stich, H.F. (1980). Enhancing and inhibiting effects of propyl gallate on carcinogen-induced mutagenesis. $\mathrm{J}$. Environ. Pathol. Toxicol. 4, 159-167.

Saito, T., Abe, D., and Sekiya, K. (2009). Flavanone exhibits PPARgamma ligand activity and enhances differentiation of 3T3-L1 adipocytes. Biochem. Biophys. Res. Commun. 380, 281-285.

Seo, J.B., Moon, H.M., Kim, W.S., Lee, Y.S., Jeong, H.W., Yoo, E.J., Ham, J., Kang, H., Park, M.G., Steffensen, K.R., et al. (2004). Activated liver $X$ receptors stimulate adipocyte differentiation through induction of peroxisome proliferatoractivated receptor gamma expression. Mol. Cell. Biol. 24, 34303444.

Sermeus, A., Cosse, J.P., Crespin, M., Mainfroid, V., de Longueville, F., Ninane, N., Raes, M., Remacle, J., and Michiels, C. (2008). Hypoxia induces protection against etoposide-induced apoptosis: molecular profiling of changes in gene expression and transcription factor activity. Mol. Cancer 7,27.

Shang, W., Yang, Y., Jiang, B., Jin, H., Zhou, L., Liu, S., and Chen, M. (2007). Ginsenoside Rb1 promotes adipogenesis in 3T3-L cells by enhancing PPARgamma2 and C/EBPalpha gene expression. Life Sci. 80, 618-625.

Spiegelman, B.M. (1998). PPAR-gamma: adipogenic regulator and thiazolidinedione receptor. Diabetes $47,507-514$

Spiegelman, B.M., and Flier, J.S. (2001). Obesity and the regulation of energy balance. Cell 104, 531-543.

Tominaga, S., Yamaguchi, T., Takahashi, S., Hirose, F., and Osumi, T. (2005). Negative regulation of adipogenesis from human mesenchymal stem cells by Jun $\mathrm{N}$-terminal kinase. Biochem. Biophys. Commun. 326, 499-504.

Wu, T.W., Fung, K.P., Zeng, L.H., Wu, J., and Nakamura, H. (1994). Propyl gallate as a hepatoprotector in vitro and in vivo. Biochem. Pharmacol. 48, 419-422. 